\title{
Aktuelle FFH-Judikatur zu Abweichungsentscheidungen und Durchsetzung
}

Walter Frenz

C Der/die Autor(en) 2020

Können Bedenken wegen erheblicher Beeinträchtigungen eines Habitatschutzgebiets nicht überwunden werden, bleibt nur eine Abweichungsentscheidung. Der EuGH betonte deren Ausnahmecharakter. Inwieweit wirkt sich dieser im Einzelnen aus? Wie verhält es sich im Streit mit der Durchsetzung des Habitatschutzes?

\section{Abweichungsentscheidungen als Ausnahme}

Die Abweichungsentscheidungen bilden die Ausnahme von dem in Art. 6 Abs. 3 FFH-RL festgelegten Genehmigungskriterium, nur Projekte zuzulassen, die das Habitatgebiet als solches nicht beeinträchtigen. Sie können daher nur zur Anwendung kommen, nachdem die Auswirkungen eines Plans oder Projekts gem. Art. 6 Abs. 3 FFHRL analysiert worden sind. ${ }^{1}$ Erst dann kann eine Abwägung dieser Gebietsbeeinträchtigungen mit zwingenden Gründen des überwiegenden öffentlichen Interesses erfolgen und die Frage beantwortet werden, ob weniger nachteilige Alternativen bestehen. ${ }^{2}$ Das gilt auch im Hinblick auf die Bestimmung der Art notwendiger Ausgleichsmaßnahmen. ${ }^{3}$

Damit geht es auch auf dieser Ebene um die sorgfältige Ermittlung der tatsächlichen Grundlagen, ob eine mögliche Beeinträchtigung der für die geschützten Gebiete wesentlichen Erhaltungsziele erfolgt, unter Berücksichtigung der bestehenden einschlägigen wissenschaftlichen Erkenntnisse. ${ }^{4}$ Dies zeigt die notwendige einheitliche Betrachtungsweise der Habitatschutzprüfung, welche sich auch auf die Abweichungsentscheidungen erstreckt. Diese unterliegen denselben fachlichen Standards wie die Ermittlung der möglichen erheblichen Beeinträchtigungen und die Prüfung von deren Verträglichkeit mit dem Schutzgebiet. Ohne eine gründliche Ermittlung und Bewertung durch die zuständige Behörde kann eine Abweichung gar nicht beurteilt werden. Dafür müssen sämtliche Gesichtspunkte des betreffenden Plans einbezogen werden. Andernfalls ist eine Abweichungsprüfung nach Art. 6 Abs. 4 FFH-RL gar nicht vorzunehmen. ${ }^{5}$

Dann bleibt ein Projekt oder Plan schon nach $\$ 34$ Abs. 2 BNatSchG unzulässig, $\$ 34$ Abs. 3 und 4 BNatSchG bleiben außer Betracht. Der Projektträger hat insoweit eine Bringschuld. Sie entspricht seiner Beweislast: So wie er nachweisen muss, dass die erheblichen Beeinträchtigungen des Schutzgebietes nicht auftreten, muss er, falls ihm dies nicht gelingt, darlegen, dass eine Abweichungsentscheidung ergehen kann.

\section{2. Überwiegende öffentliche Interessen}

\subsection{Ansatz}

Gem. $\$ 34$ Abs. 3 Nr. 1 BNatSchG kann ein Projekt aus zwingenden Gründen des überwiegenden öffentlichen Interesses einschließlich solcher sozialer oder wirtschaftlicher

Prof. Dr. Walter Frenz,

Lehrt Berg-, Umwelt- und Europarecht

an der Rheinisch-Westfälische Technische Hochschule Aachen,

Aachen, Deutschland
Art zugelassen oder durchgeführt werden. Erst auf dieser Stufe kommen also soziale und wirtschaftliche Belange zum Tragen, so dass ein Ausgleich von ökologischen, ökonomischen und sozialen Belangen im Sinne einer nachhaltigen Entwicklung erfolgt. Sie spielen nach fester Rechtsprechung weder bei der Gebietsabgrenzung ${ }^{6}$ noch bei der Erheblichkeit der Beeinträchtigungen ${ }^{7}$ noch bei der Alternativenprüfung eine Rolle. ${ }^{8}$

Das Interesse, das die Verwirklichung eines Projektes oder Planes rechtfertigen kann, muss gem. $\$ 34$ Abs. 3 Nr. 1 BNatSchG zugleich ,öffentlich “ und ,überwiegend“ sein und damit nach dem EuGH eine solche Wichtigkeit aufweisen, dass es gegen das Ziel des Habitatschutzes in Gestalt der Erhaltung der natürlichen Lebensräume sowie der wildlebenden Tiere und Pflanzen abgewogen werden kann. ${ }^{9}$ Unter solche Interessen fasst die Kommission einmal Situationen, in denen Projekte auf den Schutz von Grundwerten für das Leben der Bürger (Gesundheit, Sicherheit und Umwelt) abzielen. ${ }^{10}$ Diese Aspekte werden auch in $\$ 34$ Abs. 4 BNatSchG genannt und gelten damit erst recht für $\$ 34$ Abs. 3 BNatSchG. ${ }^{11}$ Weiter benennt die Kommission Projekte im Rahmen grundlegender Politiken für Staat und Gesellschaft oder der Durchführung von Tätigkeiten wirtschaftlicher oder sozialer Art zur Erbringung gemeinwirtschaftlicher Leistungen. ${ }^{12}$

1) EuGH, Urt. v. 17.4.2018 - C-441/17, ECLI:EU:C:2018:255, NuR 2018, 327, Rdnr. 189 (Wald von Bialowieza)

2) EuGH, Urt. v. 29.7.2019-C-411/17,ECLI:EU:C:2019:622, NuR 2019, 538, Rdnr. 150 (Inter-Environnement Wallonie (Doel)); EuGH, Urt. v. 17.4.2018 - C-441/17, ECLI:EU:C:2018:255, NuR 2018, 327, Rdnr. 191 (Wald von Bialowieza).

3) EuGH,Urt.v.29.7.2019-C-411/17,ECLI:EU:C:2019:622, NuR 2019, 538, Rdnr. 150 (Inter-Environnement Wallonie (Doel)); EuGH, Urt. v. 17.4.2018 - C-441/17, ECLI:EU:C:2018:255, NuR 2018, 327, Rdnr. 191 (Wald von Bialowieza).

4) EuGH, Urt v. 29.7.2019-C-411/17, ECLI:EU:C:2019:622 NuR 2019, 538, Rdnr. 153 (Inter-Environnement Wallonie (Doel)); EuGH, Urt. v. 17.4.2018 - C-441/17, ECLI:EU:C:2018:255, NuR 2018, 327, Rdnr. 113 (Wald von Bialowieza); EuGH, Urt. v. 26.7.2018 - C-164/17, EU:C:2018:593, Rdnr. 40 (Grace und Sweetman).

5) EuGH, Urt. v. 29.7.2019 - C-411/17, ECLI:EU:C:2019:622, NuR 2019，538，Rdnr. 154 (Inter-Environnement Wallonie (Doel)).

6) Aktuell abl. etwa BVerwG, Urt. v. 27.11.2018 - 9 A 8.17, BVerwGE 163, 380, Rdnr. 67.

7) Anders Frenz, UPR 2014, 88

8) EuGH, Urt. v. 14.1.2016 - C-399/14, ECLI:EU:C:2016:10 NuR 2016, 119, Rdnr. 77 (Grüne Liga Sachsen u. a.).

9) EuGH, Urt. v. 29.7.2019 - C-411/17, ECLI:EU:C:2019:622, NuR 2019, 538, Rdnr. 155. (Inter-Environnement Wallonie (Doel)); Urt. v. 11.9.2012 - C-43/10, ECLI:EU:C:2012:560, NuR 2012, 775, Rdnr. 121 (Nomarchiaki Aftodioikisi Aitoloakarnanias u. a.).

10) Europäische Kommission, Natura 2000 - Gebietsmanagement - Die Vorgaben des Artikels 6 der Habitat-Richtlinie 92/43/ EWG, ABl. 2019 C 33, 1, 41

11) Frenz, in: ders./Müggenborg, BNatSchG, 2. Aufl. 2016, $₫ 34$ Rdnr. 136.

12) Europäische Kommission, Natura 2000 - Gebietsmanagement - Die Vorgaben des Artikels 6 der Habitat-Richtlinie 92/43/ EWG, AB1. 2019 C 33, 1, 41. 


\subsection{Energieversorgungssicherheit als grundlegendes Unionsziel}

Besondere Bedeutung haben für den EuGH die grundlegenden Ziele der Union - so die Energieversorgungssicherheit im Energiebereich nach Art. 194 Abs. 1 lit. b) AEUV. ${ }^{13}$ Dabei ist grundsätzlich eine gleichgewichtige Abwägung vorzunehmen, sind doch die Ziele im Ausgangspunkt als gleichrangig zu betrachten (Art. 7 AEUV). ${ }^{19}$ In der konkreten Einzelfallbetrachtung müssen dann die Aspekte, welche für das Vorhaben sprechen, überwiegen.

Die Energieversorgungssicherheit benennt der EuGH explizit auf der Basis von Art. 194 Abs. 1 lit. b) AEUV im Hinblick auf Kernkraftwerke. ${ }^{15}$ Hier zeigt sich die Energieneutralität des Unionsrechts. Relevant wird sie etwa, wenn es um die Genehmigung von Kernkraftwerken in anderen EU-Staaten mit möglichen Auswirkungen aufDeutschland geht.

\section{3 Öffentliche vs. private Belange?}

Allerdings werden gerade Kraftwerke zumeist von privaten Investoren getragen. Der EuGH differenzierte insoweit nicht. Der Begriff des öffentlichen Interesses schließt nur scheinbar private Interessen aus. Zwar kommen rein private Interessen nicht in Betracht ${ }^{16}$ vielmehr muss ein Projekt privater Natur seinem Wesen nach als auch aufgrund seines wirtschaftlichen und sozialen Kontextes tatsächlich von überwiegendem öffentlichen Interesse sein. ${ }^{17}$ Vielfach fällt aber privates und öffentliches Interesse zusammen, so wenn es um zentrale Infrastrukturprojekte wie Flughäfen und Abfallentsorgungsanlagen ${ }^{18}$ oder den Abbau von Rohstoffen geht. Es genügt aber regelmäßig nicht die Infrastruktur für die bloße Erweiterung oder Ansiedlung eines Unternehmens. ${ }^{19}$

\subsection{Deutliches Überwiegen ohne erforderliche Sachzwänge}

Also müssen die relevanten Gründe des öffentlichen Interesses die betroffenen Belange des Habitatschutzes überwiegen ${ }^{20}$, und zwar deutlich. Ansonsten handelt es sich um keine zwingenden Gründe, die das Projekt notwendig machen. Ein leichtes Überwiegen reicht daher nicht aus. Allerdings bedarf es auch keiner Sachzwänge, denen niemand ausweichen kann. ${ }^{21}$ Vielmehr genügt entsprechend der Formulierung in Art. 6 Abs. 4 FFH-RL ein ,durch Vernunft und Verantwortungsbewusstsein geleitetes staatliches Handeln“, wie das BVerwG wiederum in der ElbvertiefungsEntscheidung formulierte. ${ }^{22}$

\subsection{Verstärktes Gewicht bei prioritären Arten und Lebensräumen}

Sind in einem Gebiet prioritäre Arten vorhanden oder handelt es sich um einen prioritären natürlichen Lebensraumtyp, kann nur die Notwendigkeit der Abwendung einer tatsächlichen und schwerwiegenden Gefahr, beispielsweise dass die Stromversorgung des betreffenden Staates unterbrochen wird, einen Grund der öffentlichen Sicherheit nach Art. 6 Abs. 4 UAbs. 2 FFH-RL darstellen. ${ }^{23}$ Daran zeigt sich das notwendige verstärkte Gewicht bei einem Vorkommen prioritärer Arten oder Lebensraumtypen. Zudem stellt der EuGH auf die öffentliche Sicherheit $a b$ und ordnet die Energieversorgung nicht als allgemeinen wirtschaftlichen Aspekt. Die Frage, ob und inwieweit über die in Art. 6 Abs. 4 FFH-RL und in $\$ 34$ Abs. 4 BNatSchG genannten Aspekte der Gesundheit des Menschen, der öffentlichen Sicherheit oder der maßgeblich günstigen Auswirkungen auf die Umwelt auch andere Gründe einzubeziehen sind, die allerdings nur nach Vorlage bei der Kommission relevant sein können, war daher nicht von Bedeutung. Immerhin betonte der EuGH die höhere Schwelle und liegt damit auf der Linie des BVerwG, dass ,nur eine begrenzte Zahl solcher zwingender Gründe" geeignet ist, die Beein- trächtigung prioritärer Lebensraumtypen oder Arten zu rechtfertigen - nicht indes Gemeinwohlbelange minderen Gewichts wie freizeitbedingte Bedürfnisse der Bevölkerung $^{24}$ oder auch wirtschaftliche Allgemeinbelange. ${ }^{25}$

So bleibt auch die Aufführung bestimmter Elemente in Art. 6 Abs. 4 UAbs. 2 FFH-RL und in $\$ 34$ Abs. 4 S. 1 BNatSchG gewahrt, wo die Interessen sozialer oder wirtschaftlicher Art nicht genannt sind. Daher können nur vereinzelte Gründe auch nach $\$ 34$ Abs. 4 S. 2 BNatSchG geltend gemacht werden. Es muss sich dabei aber um Einzelkonstellationen handeln. Es wird nur für spezielle und damit nur ausnahmsweise auftretende Fälle der Weg über die Kommission geöffnet. Diese mit sämtlichen Projekten, welche öffentliche Interessen sozialer oder wirtschaftlicher Art verfolgen, zu befassen, würde zu einer starken Arbeitsüberlastung führen. ${ }^{26}$

Das BVerwG ist gleichwohl teilweise weiter. Es nimmt als zwingenden Grund die Planrechtfertigung jedenfalls dann hinzu, wenn das Vorhaben gesetzlich vorgesehen ist und zum transeuropäischen Verkehrsnetz gehört. ${ }^{27}$ Dabei soll die Summe der genannten Gründe zählen. ${ }^{28}$ Damit wird aber die bewusste Verengung in $\$ 34$ Abs. 4 S. 1 BNatSchG umgangen ${ }^{29}$. Dabei können unabhängig von dieser Konzeption des BVerwG gerade im Verkehrsbereich Rechtfertigungsansätze gewonnen werden: Eine dort angestrebte Entlastungsfunktion und Sicherheitssteigerung ${ }^{30}$ dient $\mathrm{zu}-$ gleich günstigen Umweltauswirkungen sowie der Gesundheit des Menschen nach $\$ 34$ Abs. 4 S. 1 BNatSchG. ${ }^{31}$

13) EuGH, Urt. v. 29.7.2019 - C-411/17, ECLI:EU:C:2019:622, NuR 2019, 538, Rdnr. 156 (Inter-Environnement Wallonie (Doel)).

14) Näher Frenz, in: ders./Müggenborg/Cosack/Hennig/Schomerus, EEG, 5. Aufl. 2018, \$1 Rdnr. 66 ff. m. w. N.

15) EuGH, Urt. v. 29.7.2019-C-411/17, ECLI:EU:C:2019:622, NuR 2019, 538, Rdnr. 156 (Inter-Environnement Wallonie (Doel)).

16) Möckel, in: Schlacke (Hrsg.), GK-BNatSchG, 2. Aufl. 2016, \$34 Rdnr. 142.

17) EuGH, Urt. v. 16.2.2012 - C-182/10, ECLI:EU:C:2012:82, NuR 2012,703, Rdnr. 77 (Solvay).

18) BVerwG, Beschl. v. 3.6.2010 - 4 B 54.09, NVwZ 2010, 1289 ff.; Gellermann, in: Landmann/Rohmer, Umweltrecht, Stand: 2/2019, §34 BNatSchG Rdnr. 38; Möckel, in: Schlacke (Hrsg.), GK-BNatSchG, 2. Aufl. 2016, \$34 Rdnr. 144.

19) EuGH, Urt. v. 16.2.2012 - C-182/10, ECLI:EU:C:2012:82, NuR 2012, 703, Rdnr. 76 (Solvay).

20) Siehe etwa OVG Lüneburg, Urt. v. 20.5.2009 - 7 KS 28/07, NuR 2009, 719, Ziff. 2.3.2.2.3; Jarass, NuR 2007, 371, $376 \mathrm{f}$.

21) BVerwG, Urt. v. 9.2.2017 - 7 A 2.15, BVerwGE 158, 1, NuR 2017, 552, Rdnr. 389 (Elbvertiefung); Spieth/Appel, NuR 2009, 669,671 .

22) BVerwG, Urt. v. 9.2.2017 - 7 A 2.15, BVerwGE 158, 1, NuR 2017, 552, Rdnr. 389 (Elbvertiefung); bereits BVerwG, Urt. v. 12.3.2008 - 9 A 3/06, BVerwGE 130, 299, NuR 2008, 633, Rdnr. 153; OVG Koblenz, Urt. v. 8.11.2007 - 8 C 523/06, NuR 2008, 181, 187.

23) EuGH, Urt. v. 29.7.2019 - C-411/17, ECLI:EU:C:2019:622, NuR 2019, 538, Rdnr. 158 (Inter-Environnement Wallonie (Doel)).

24) BVerwG, Urt. v. 23.4.2014 - 9 A 25.12, BVerwGE 149, 289 NuR 2014, 706, Rdnr. 73 (A 49 unter Verweis auf EuGH), Urt. v. 14.4.2005 - C-441/03, ECLI:EU:C:2005:233, NuR 2005, 450, Rdnr. 22.

25) Gellermann, in: Landmann/Rohmer, Umweltrecht, Stand: 2/2019, §34 BNatSchG Rdnr. 47.

26) Frenz, in: ders./Müggenborg, BNatSchG, 2. Aufl. 2016, \$34 Rdnr. 145.

27) BVerwG, Urt. v. 23.4.2014 - 9 A 25.12, BVerwGE 149, 289, NuR 2014, 706, Rdnr. 74 (A 49).

28) BVerwG, Urt. v. 23.4.2014 - 9 A 25.12, BVerwGE 149, 289 , NuR 2014, 706, Rdnr. 74 (A 49).

29) Siehe auch: Günes/Fisahn, EurUP 2007, 220, 227.

30) BVerwG, Urt. v. 23.4.2014 - 9 A 25.12, BVerwGE 149, 289, NuR 2014, 706, Rdnr. 75 (A 49).

31) Frenz, in: ders./Müggenborg, BNatSchG, 2. Aufl. 2016, §34 Rdnr. 146. 


\section{Alternativenprüfung}

Die Alternativenprüfung war einer der Schwerpunkte der Elbvertiefungs-Entscheidung des BVerwG. \34 Abs. 3 Nr. 2 BNatSchG verlangt sie in Umsetzung von Art. 6 Abs. 4 UAbs. 1 FFH-RL. Auch insoweit geht es um eine umfassende Prüfung sämtlicher Gesichtspunkte auf der Basis der besten einschlägigen wissenschaftlichen Erkenntnisse. ${ }^{32}$ Das betrifft allerdings die notwendige Darlegung der Anlagenkonzeption und ihrer Auswirkungen.

Daraus folgt nicht etwa eine habitatschutzbezogene Alternativenprüfung dergestalt, dass möglichst viele Alternativen einzubeziehen wären. Ausgangspunkt dafür ist vielmehr die Projektkonzeption des Vorhabenträgers. Gerade im Rahmen der Abweichungsentscheidung müssen die ökonomischen Belange entsprechend der das gesamte Unionsrecht überwölbenden Nachhaltigkeitskonzeption nach Art. 3 Abs. 3 EUV $^{33}$ adäquat und in dem ihnen eigenen Gehalt zur Geltung kommen können, wenn sie schon nach der Rechtsprechung auf den vorhergehenden Prüfungsstufen praktisch unbeachtlich sind.

Der Vorhabenträger muss sich daher nicht auf ein anderes Projekt verweisen lassen. Das zeigt die Elbvertiefungs-Entscheidung des BVerwG: Das Vorhaben sollte den Verkehrsbedarf für eine bessere tideunabhängige und tideabhängige Erreichbarkeit des Hamburger Hafens vor allem mit Containerschiffen decken; dieses Ziel ist durch eine Kooperation mit anderen Häfen gar nicht zu erreichen. Eine solche „Konzeptalternative“ bildet daher ein aliud mit anderen und nicht identischen Planungszielen. ${ }^{34}$ Hafen ist nicht gleich Hafen. Jeder Standort ist mit einer spezifischen Lage und einer damit einhergehenden Infrastruktur verknüpft. So ermöglichen die Hamburger Anlagen und Vorrichtungen für Schiffsarbeiten etc. eine effektive Abfertigung; in Wilhelmshaven bedürfte es insoweit noch eines erheblichen Ausbaus. ${ }^{35}$ Eine Kombination beider bildet ein gänzlich anderes Konzept, das die Containerschiffe selbst gar nicht bis zum Hamburger Hafen führt.

Mit dem Vorprojekt verfolgte selbstständige Teilziele brauchen ebenfalls nicht aufgegeben zu werden. Es müssen nur gewisse Abstriche hingenommen werden, sofern sich dann geringere Beeinträchtigungen für das fragliche Habitatschutzgebiet und dessen Erhaltungsziele ergeben. ${ }^{36}$ Diese Abstriche gehen aber nicht so weit, dass wesentliche Projektziele aufgegeben werden müssen - so eine bestimmte Ausbautiefe und ein bestimmter Ausbauumfang für einen bestimmten Verkehrsbedarf, und zwar auch, um die Funktions- und Wettbewerbsfähigkeit eines Infrastrukturprojekts zu stärken, so für den Hamburger Hafen, woraus sich eine bestimmte Ausbautiefe ergab. ${ }^{37}$ Der Projektträger muss sich nicht mit einem Mindestausbau oder mit Minimalvarianten zufrieden geben - so bei einem Hafen mit einer geringeren Ausbautiefe oder mit einem Teilverzicht auf einen Ausbau für die tideunabhängige oder die tideabhängige Fahrt oder mit einer durchgängigen Halbierung der Tiefgangsziele (,Elbe light“): Insoweit handelt es sich nicht um zumutbare Alternativen, wie das BVerwG klar entschied. ${ }^{38}$

Eine Alternative muss weiter verhältnismäßig sein. Die Vermeidungsanstrengungen sind daher ins Verhältnis zu dem damit erreichbaren Gewinn für Natur und Umwelt zu setzen. Maßgeblich ist dabei die Funktion des durch Art. 4 FFH-RL begründeten Schutzregimes, so dass nur gewichtige naturschutzexterne Gründe die Möglichkeit einer Alternativlösung auszuschließen vermögen, mithin eine Projektverwirklichung zulasten des Integritätsinteresses des durch Art. 4 FFH-RL festgelegten kohärenten Systems rechtfertigen. ${ }^{39}$ Auch insoweit sind also ökologische Gesichtspunkte hereinzunehmen. Mit ihnen sind die ökonomischen Belange abzuwägen. Letztere dürfen indes nicht zu kurz kommen, auch wenn der Umstand adäquat $\mathrm{zu}$ würdigen ist, dass sie im Kontext einer habitatschutzrechtlichen Prüfung auftreten. Gleichwohl ist der grund- sätzliche Gleichrang von ökologischen und ökonomischen Belangen zu wahren.

Im Bereich des Habitatschutzes ist freilich auch bei dieser Abwägung besonders auf den Erhalt der geschützten Arten und Lebensräume zu achten. Dementsprechend dürfen wirtschaftliche Kosten nicht allein bestimmend sein, wie der EuGH in seiner Dresdner Waldschlösschenbrücke-Entscheidung spezifisch zur Alternativenprüfung ausführte: Diesen Kosten darf nicht die gleiche Bedeutung zukommen wie dem mit der FFH-RL verfolgten Ziel der Erhaltung der natürlichen Lebensräume sowie der wildlebenden Tiere und Pflanzen. ${ }^{40}$ Das betrifft aber nur das Gewicht, nicht die Relevanz als solche. So musste bei der Frage, ob als Alternative für die Elbvertiefung zum Ausbau des Hamburger Hafens auch eine Kombilösung mit dem JadeWeserPort in Wilhelmshaven in Betracht kommt, ebenfalls der dann notwendige Umzug bzw. Ausbau der Hafeninfrastruktur für Schiffsarbeiten berücksichtigt werden. Es zählen also auch Kostengründe ${ }^{41}$ - aber eben in dem Rahmen, in dem sie zum Zuge kommen können, ohne ein Habitatschutzgebiet schwerwiegend zu tangieren.

Daher war die Sicherung von Arbeitsplätzen auch für das BVerwG ein wichtiger Aspekt im Rahmen der Elbvertiefung, wobei genaue Prognosen zur Zahl naturgemäß mit erheblichen Unsicherheiten verbunden sind; das ist aber angesichts der Abhängigkeit von der weltwirtschaftlichen Entwicklung unschädlich, solange die langfristigen Wachstumspotenziale und die dauerhafte Sicherung von Arbeitsplätzen deutlich werden. ${ }^{42}$ Dem Vorhabenträger müssen auch langfristige Planungen möglich sein. Nur so lassen sich gerade die wichtigen Wirtschaftsprojekte, welche in der Lage sind, auch in der Abwägung mit Habitatschutzbelangen standzuhalten, weiterhin verwirklichen - ggf. gerade auch im Interesse des Habitatschutzes: Erst eine gröBere Vertiefung der Fahrrinne verhindert, dass bereits nach wenigen Jahren erneut ein weiterer Aushub erfolgen muss, der wiederum einen Eingriff in ein Habitatschutzgebiet mit sich bringt, kaum dass die Folgen des früheren Ausbaus verwachsen sind. ${ }^{43}$

\section{Ausgleichsmaßnahmen}

Auch Ausgleichsmaßnahmen werden von der Pflicht zur sorgfältigen Ermittlung und Bewertung der Beeinträchtigungen eines geschützten Gebietes erfasst: Sie sollen die Beeinträchtigungen, die von dem zugelassenen bzw.

32) EuGH, Urt. v. 29.7.2019 - C-411/17, ECLI:EU:C:2019:622, NuR 2019, 538, Rdnr. 150, 153 (Inter-Environnement Wallonie (Doel))

33) Frenz, Handbuch Europarecht, Bd. 6, 2011, Rdnr. $2086 \mathrm{ff}$.

34) BVerwG, Urt. v. 9.2.2017 - 7 A 2.15, BVerwGE 158, 1, NuR 2017, 552, Rdnr. 412 (Elbvertiefung); A.A. Feldt/Schumacher, $\mathrm{NuR} 2015,391,392 \mathrm{ff}$

35) Frenz, in: ders./Müggenborg, BNatSchG, 2. Aufl. 2016, \$34 Rdnr. 116 ff. auch für das Folgende. Näher bereits Frenz, NuR 2015, 683, 684

36) BVerwG, Urt. v. 17.1.2007 - 9 A 20.05, BVerwGE 128, 1, Rdnr. 143, NuR 2007, 336 (Westumfahrung Halle).

37) BVerwG, Urt. v. 9.2.2017 - 7 A 2.15, BVerwGE 158, 1, NuR 2017, 552, Rdnr. 415 (Elbvertiefung)

38) BVerwG, Urt. v. 9.2.2017 - 7 A 2.15, BVerwGE 158, 1, NuR 2017, 552, Rdnr. 414 (Elbvertiefung)

39) BVerwG, Urt. v. 23.4.2014 - 9 A 25.12, BVerwGE 149, 289 , NuR 2014, 706, Rdnr. 78 (A 49)

40) EuGH, Urt. v. 14.1.2016 - C-399/14, ECLI:EU:C:2016:10, NuR 2016, 119, Rdnr. 77 (Grüne Liga Sachsen u.a.).

41) BVerwG, Urt. v. 23.4.2014 - 9 A 25.12, BVerwGE 149, 289 , NuR 2014, 706, Rdnr. 78 (A 49).

42) BVerwG, Urt. v. 9.2.2017 - 7 A 2.15, BVerwGE 158, 1, NuR 2017, 552, Rdnr. 402f. (Elbvertiefung).

43) Siehe BVerwG, Urt. v. 9.2.2017 - 7 A 2.15, BVerwGE 158, 1 , $\mathrm{NuR}$ 2017, 552, Rdnr. 415 (Elbvertiefung). 
durchgeführten Projekt ausgehen und durch die Abweichungsentscheidung nach $₫ 34$ Abs. 3 (i. V.m. Abs. 4) BNatSchG hingenommen werden, aufwiegen, allerdings nur im Hinblick auf die Kohärenz des Netzes „Natura 2000“.44 Dessen globale Kohärenz gilt es angesichts der Bedeutung von Natura 2000 sorgfältig zu sichern. Das erfolgt durch eine genaue Ermittlung der Gebietsbeeinträchtigungen, um die Art etwaiger Ausgleichsmaßnahmen ermitteln zu können. ${ }^{45}$

Sie bilden entsprechend dem aktuellen Leitfaden der Kommission das letzte Mittel, ${ }^{46}$ nach dem bisherigen Leitfaden den letzten Ausweg nach genauer Feststellung der negativen Auswirkungen auf die Integrität eines zum Netz „Natura 2000“ gehörenden Gebiets. ${ }^{47}$ Diese negativen Auswirkungen sollen sie gezielt ausgleichen, wenn die anderen FFH-Schutzvorschriften vollständig berücksichtigt wurden und entschieden wurde, ein Projekt oder einen Plan trotz zumindest nicht ausschließbarer negativer Auswirkungen auf die Integrität eines Natura 2000-Gebiets durchzuführen. ${ }^{48}$ Dieser Ausgleich soll genau den negativen Auswirkungen auf den betroffenen Lebensraum entsprechen. ${ }^{49}$ Bei einer Beeinträchtigung selbst prioritärer Lebensräume kann die Neuanlage und Verbesserung von Auenwäldern sowie die Renaturierung und Optimierung von Flussläufen erfolgen. ${ }^{50}$

Formal geht es nicht um die eigentliche Zulässigkeit des Projektes in dem beeinträchtigten FFH-Gebiet, sondern um die Folgen der mit der Zulassung in Kauf genommenen Beeinträchtigungen und damit um eine Rechtsfolge. ${ }^{51}$ Diese ist allerdings zwingend ${ }^{52}$ und zugleich Voraussetzung für die Zulassung des Projekts, weshalb auch von einer Tatbestandsvoraussetzung ausgegangen wird. ${ }^{53}$ Dies entspricht der früheren Einstufung durch das BVerwG. ${ }^{54}$ De facto bildet die Kompensation eine solche Voraussetzung insoweit, als das Projekt bzw. der Plan anders nicht zugelassen werden darf. Es handelt sich um ein ,nicht überwindbares Zulassungshindernis“. 55 Gleichwohl verlangt das BVerwG zu Recht nicht den vollen Nachweis der Wirksamkeit wie bei Schadensvermeidungs- und Schadensminderungsmaßnahmen, sondern nur eine hohe Wahrscheinlichkeit der Wirksamkeit nach aktuellem wissenschaftlichen Erkenntnisstand. ${ }^{56}$ Zwar widerspricht dies der engen Verbindung mit den anderen Absätzen, ist aber unweigerlich Konsequenz der prognostischen Beurteilung.

\section{Wirksame Durchsetzung}

Die Durchsetzung der FFH-Standards muss wirksam gewährleistet sein. Das hat der EuGH erst wieder in seiner Doel-Entscheidung ${ }^{57}$ betont. Es ging dort um die Durchsetzung der Umweltstandards nach der UVP-RL und der FFH-RL auf Projekte, welche schon genehmigt sind, aber diesen Standards nicht entsprechen. Generell besteht der Grundsatz der loyalen Zusammenarbeit nach Art. 4 Abs. 3 EUV. ${ }^{58}$ Nach den Grundsätzen der Äquivalenz und der Effektivität müssen die Mitgliedstaaten einschließlich der nationalen Gerichte trotz der mitgliedstaatlichen Verfahrensautonomie die unionsrechtlichen Standards gleichheitsgerecht und praktisch realisierbar durchsetzen. ${ }^{59}$ Nur ausnahmsweise kann es nationalen Stellen gestattet sein, bestimmte Wirkungen eines für nichtig erklärten Rechtsaktes des Staates aufrechtzuerhalten. ${ }^{60}$ Daher musste auch für die Waldschlösschenbrücke in Dresden trotz Genehmigung vor Ausweisung eines Habitatschutzgebietes, aber einer Realisierung danach selbst ein Abriss in Betracht gezogen werden; jedenfalls mussten die FFH-Standards in vollem Umfang durchgesetzt werden. ${ }^{61}$

Eine solche Konstellation muss aber die absolute Ausnahme bleiben, weil ansonsten die einheitliche Anwendung des Unionsrechts beeinträchtigt würde, sofern nationale Gerichte befugt wären, auch nur vorübergehend nationa- len Bestimmungen Vorrang vor dem Unionsrecht einzuräumen. ${ }^{62}$ Hier kommt eine solche Durchbrechung nach dem $\mathrm{EuGH}$, der als einziger die Voraussetzungen festlegen kann, nur dann infrage, wenn bei Aufhebung oder Aussetzungen der Wirkungen einer Maßnahme die tatsächliche und schwerwiegende Gefahr einer Unterbrechung der Stromversorgung des betreffenden Mitgliedstaats bestünde, der nicht mit anderen Mitteln und Alternativen, insbesondere im Rahmen des Binnenmarkts, entgegengetreten werden kann. ${ }^{63}$ Und auch dies gilt nur für den absolut notwendigen Zeitpunkt, um dann die Rechtswidrigkeit des bisheri-

44) Bereits EuGH, Urt. v. 20.9.2007 - C-304/05, ECLI:EU:C 2007:532, NuR 2007, 679, Rdnr. 18 (Kommission/Italien); EuGH, Urt. v. 17.4.2018 - C-441/17, ECLI:EU:C:2018:255, NuR 2018, 327, Rdnr. 190 (Wald von Bialowieza).

45) EuGH, Urt. v. 29.7.2019 - C-411/17, ECLI:EU:C:2019:622, NuR 2019, 538, Rdnr. 150 (Inter-Environnement Wallonie (Doel)).

46) Europäische Kommission, Natura 2000 - Gebietsmanagement - Die Vorgaben des Artikels 6 der Habitat-Richtlinie 92/43/ EWG, ABl. 2019 C 33, 1, 42.

47) Leitfaden der Kommission zu Art. 6 Abs. 4 der „Habitatrichtlinie“ 92/43/EG, Januar 2007, 12

48) Europäische Kommission, Natura 2000 - Gebietsmanagement - Die Vorgaben des Artikels 6 der Habitat-Richtlinie 92/43/ EWG, ABl. 2019 C 33, 1, 42.

49) Europäische Kommission, Natura 2000 - Gebietsmanagement - Die Vorgaben des Artikels 6 der Habitat-Richtlinie 92/43/ EWG, ABl. 2019 C 33, 1, 42.

50) Europäische Kommission, Natura 2000 - Gebietsmanagement - Die Vorgaben des Artikels 6 der Habitat-Richtlinie 92/43/ EWG, ABl. 2019 C 33, 1, 42.

51) So BVerwG, Urt. v. 12.3.2008 - 9 A 3.06, BVerwGE 130, 299 , NuR 2008, 633, Rdnr. 197; aus der Lit. Durner, NuR 2001, 601, 609; Jarass, NuR 2007, 371, 379; Spieth/Appel, NuR 2009, 669, 674

52) Gellermann, in: Landmann/Rohmer, Umweltrecht, Stand: 2/2019, §34 BNatSchG Rdnr. 52.

53) VGH Kassel, Urt. v. 2.1.2009 - 11 B 368/08.T, NuR 2009, 255, 275; Köck, ZUR 2005, 466, 469; Storost, DVB1. 2009, 673, 677; Lüttgau/Kockler, in: BeckOK Umweltrecht, Stand: 07/2019, $\$ 34$ BNatSchG Rdnr. 24; Möckel, in: Schlacke (Hrsg.), GKBNatSchG, 2. Aufl. 2016, \$34 Rdnr. 182; J. Schumacher/A. Schumacher, in: Schumacher/Fischer-Hüftle, BNatSchG, 2. Aufl. 2010, \$34 Rdnr. 106; Steeck/Lau, NVwZ 2009, 616, 621. A. A. Durner, NuR 2001, 601, 609, Jarass, NuR 2007, 371, 379; Spieth/ Appel, NuR 2009, 669, 674.

54) BVerwG, Urt. v. 17.1.2007 - 9 A 20.05, BVerwGE 128, 1, NuR 2007, 336, Rdnr. 148 (Westumfahrung Halle).

55) Gellermann, in: Landmann/Rohmer, Umweltrecht, Stand: 2/2019, \$34 BNatSchG Rdnr. 52.

56) BVerwG, Urt. v. 6.11.2012 - 9 A 17.11, BVerwGE 145, 40, NuR 2014, 344, Rdnr. 83 - A 33; BVerwG, Urt. v. 9.2.2017 - 7 A 2.15, BVerwGE 158, 1, NuR 2017, 552, Rdnr. 420 (Elbvertiefung)

57) EuGH, Urt. v. 29.7.2019 - C-411/17, ECLI:EU:C:2019:622, NuR 2019, 538 (Inter-Environnement Wallonie (Doel)).

58) EuGH, Urt. v. 29.7.2019 - C-411/17, ECLI:EU:C:2019:622, NuR 2019, 538, Rdnr. 170 (Inter-Environnement Wallonie (Doel)).

59) EuGH, Urt. v. 28.2.2012 - C-41/11, ECLI:EU:C:2012:103, NuR 2012, 254, Rdnr. 45 m. w. N. (Inter-Environnement Wallonie und Terre wallonne).

60) EuGH, Urt. vom 29.7.2019 - C-411/17, ECLI:EU:C:2019:622, NuR 2019, 538, Rdnr. 178 (Inter-Environnement Wallonie (Doel)).

61) EuGH, Urt. v. 14.1.2016 - C-399/14, ECLI:EU:C:2016:10, NuR 2016, 119, Rdnr. 77 (Grüne Liga Sachsen u. a.) Näher dazu Frenz, NuR 2020, 94.

62) EuGH, Urt. vom 29.7.2019, C-411/17, ECLI:EU:C:2019:622, NuR 2019, 538, Rdnr. 177 (Inter-Environnement Wallonie (Doel)).

63) EuGH, Urt. v. 29.7.2019 - C-411/17, ECLI:EU:C:2019:622, NuR 2019, 538, Rdnr. 179 a. E. (Inter-Environnement Wallonie (Doel)). 
gen nationalen Rechts zu beheben. ${ }^{64}$ Angesichts des hohen Maßes des angebotenen Stroms in der EU dürfte dies aber kaum in Betracht kommen. Das gilt allerdings nicht nur für Kernkraftwerke, sondern auch für Windenergieanlagen, die unter Missachtung von FFH-Regeln genehmigt wurden.

\section{Fazit}

Auch Abweichungsentscheidungen sowie die Durchsetzung sind vom zentralen Zweck des Habitatschutzrechts geprägt, die Schutzgebiete als solche zu erhalten. Daher bildet die Grundlage wie auch bei den vorherigen Stufen der Habitatschutzprüfung die Ermittlung sämtlicher Gesichtspunkte des betreffenden Plans oder Projekts; das gilt bis zu Ausgleichsmaßnahmen, für die eine hohe Wahrscheinlichkeit ihrer Wirksamkeit genügt. Dennoch müssen ökonomische Gesichtspunkte adäquat zur Geltung gelangen. Das betrifft die möglichen Gründe für eine Abweichung vom Habitatschutz, die freilich deutlich überwiegen und näher dargetan sowie belegt sein müssen. Vor allem gilt dies bei der Prüfung von Alternativlösungen, bei welcher die Konzeption des Vorhabenträgers der Ausgangspunkt und Prüfungsmaßstab sein muss - ihm darf kein aliud aufgezwungen werden, mit dem er seine (Teil)Ziele nicht erreichen kann.
Open Access. Dieser Artikel wird unter der Creative Commons Namensnennung 4.0 International Lizenz veröffentlicht, welche die Nutzung, Vervielfältigung, Bearbeitung, Verbreitung und Wiedergabe in jeglichem Medium und Format erlaubt, sofern Sie den/die ursprünglichen Autor(en) und die Quelle ordnungsgemäß nennen, einen Link zur Creative Commons Lizenz beifügen und angeben, ob Änderungen vorgenommen wurden.

Die in diesem Artikel enthaltenen Bilder und sonstiges Drittmaterial unterliegen ebenfalls der genannten Creative Commons Lizenz, sofern sich aus der Abbildungslegende nichts anderes ergibt. Sofern das betreffende Material nicht unter der genannten Creative Commons Lizenz steht und die betreffende Handlung nicht nach gesetzlichen Vorschriften erlaubt ist, ist für die oben aufgeführten Weiterverwendungen des Materials die Einwilligung des jeweiligen Rechteinhabers einzuholen.

Weitere Details zur Lizenz entnehmen Sie bitte der Lizenzinformation auf http://creativecommons.org/licenses/by/4.0/deed.de.

Open access funding provided by Projekt DEAL.

64) EuGH, Urt. vom 29.7.2019 - C-411/17, ECLI:EU:C:2019:622, NuR 2019, 538, Rdnr. 181 (Inter-Environnement Wallonie (Doel)).

\section{Allgemeiner Rahmen der energetischen Folgenutzung von ehemaligen Kohlestandorten im Zuge des Kohleausstiegs*}

\section{Anna Wehmeyer}

\author{
(c) Der/die Autor(en) 2020
}

Dieser Beitrag befasst sich mit der Frage, unter welchen Bedingungen die ehemals bergbaulich und zum Zweck der Kohleverstromung genutzten Flächen zum erforderlichen Ausbau der erneuerbaren Energien und Energiezwischenspeicher verwendet werden können. Dazu wird untersucht, welche Empfehlungen der Abschlussbericht der Kommission „Wachstum, Strukturwandel und Beschäftigung" zur Folgenutzung ehemaliger Kohlestandorte enthält und danach, welche rechtlichen Bestimmungen sich durch das Bundesberggesetz (BBergG), das Raumordnungsrecht und das Genehmigungsrecht für erneuerbare Energien und Energiezwischenspeicher ergeben. Außerdem wird der aktuelle Stand in Form von aktuellen Projekten und die aktuelle Gesetzgebung diesbezüglich betrachtet. Der erste Teil untersucht den Abschlussbericht der Kommission „Wachstum, Strukturwandel und Beschäftigung" und die allgemeinen rechtlichen Rahmenbedingungen, denen die Folgenutzung der ehemaligen Kohlestandorte und die Errichtung von Anlagen zur Erzeugung von Strom aus erneuerbaren Energien unterstehen.

\section{Einleitung}

In ihrem Abschlussbericht hat die Kommission „Wachstum, Strukturwandel und Beschäftigung“" als Enddatum für die Kohleverstromung und damit auch für den Braunkohleabbau das Jahr 2038 festgelegt. Der Ausstieg soll schrittweise geschehen.

Die Ziele zum Ausbau der Erneuerbaren Energien sind in $\$ 1$ Erneuerbare-Energien-Gesetz mit Anteilen von $40 \%$ bis $45 \%$ bis zum Jahr 2025, $55 \%$ bis $60 \%$ bis zum Jahr 2035

Anna Wehmeyer, RWTH Aachen University,

Aachen, Deutschland und mindestens $80 \%$ bis zum Jahr 2050 am Bruttostromverbrauch festgehalten. In Deutschland gibt es aber nur sehr wenige Flächen, die nicht entweder bereits in Benutzung sind oder für die nicht eine anderweitige Nutzung vorgesehen ist. Durch den Kohleausstieg werden sukzessive Flächen wieder ,frei“".

Für diese Flächen wird untersucht, welche Rahmenbedingungen für eine Folgenutzung zur Errichtung von Anlagen zur Erzeugung von Strom aus Erneuerbaren Energien vorliegen. Berücksichtigt werden zunächst die Windenergie und die Solarenergie, da die Windenergie mit 25\% und die Solarenergie mit 9\% in 2019 den höchsten Anteil der Erneuerbaren Energien (gesamt 46,1\%) an der deutschen Nettostromerzeugung hatten. ${ }^{1}$ Der Anteil der Biomasse war mit 8,6\% ungefähr so hoch wie der der Solarenergie, aber durch die aktuellen Bestrebungen, Biomasse in bereits vorhandenen Kohlekraftwerken mitzuverbrennen, ${ }^{2}$ ergibt sich diesbezüglich kein Bedarf zur Betrachtung des aktuellen Stands. Weiterhin wird die Geothermie berücksichtigt, da das in Steinkohlebergwerken entstandene Grubenwasser als Wärmeträgermedium verwendet werden kann. Zusätzlich sind durch den Bergbau bereits tiefe Bohrungen vorhanden und die genaue Geologie vor Ort bekannt.

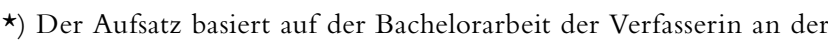
RWTH Aachen University.

1) Fraunhofer: Nettostromerzeugung in Deutschland 2019, Stand 5.1.2020, abrufbar unter https://www.energy-charts.de/energy_ pie_de.htm? year $=2019$.

2) Uniper: Biomasse-Umwandlungstechnologien für Kraftwerke, Stand 3.1.2020, abrufbar unter https://www.uniper.energy/services/de/biomasse-umwandlungstechnologien-kraftwerke. 